\title{
Macbeth, tragédie et confusion des signes
}

Jean-Paul Debax

\section{(2) OpenEdition \\ Journals}

Édition électronique

URL : http://journals.openedition.org/shakespeare/1327

DOI : 10.4000/shakespeare.1327

ISSN : 2271-6424

Éditeur

Société Française Shakespeare

Édition imprimée

Date de publication : 1 novembre 1989

Pagination : 225-240

Référence électronique

Jean-Paul Debax, "Macbeth, tragédie et confusion des signes 》, Actes des congrès de la Société française Shakespeare [En ligne], 7| 1989, mis en ligne le 01 janvier 2007, consulté le 02 mai 2019. URL : http://journals.openedition.org/shakespeare/1327 ; DOI : 10.4000/shakespeare.1327 


\title{
Société Française Shakespeare
}

\author{
Actes des Congrès \\ 1985 - 1986 - 1987
}

\section{LA FOLIE}

\section{SHAKESPEARE ET LES ARTS \\ LE TRAGIQUE}




\section{MACBETH \\ TRAGEDIE ET CONFUSION DES SIGNES}

J.P. DEBAX

Cet essai a été en partie provoqué par certaines remarques et suggestions faites au cours du colloque qui s'est tenu à Toulouse les 13 et 14 novembre 1987 sur Macbeth et The Man of Mode. Il doit donc beaucoup aux articles qui seront publiés dans le volume Miroirs de l' être qui rassemble les communications au congrès de Toulouse, même si cette dette n'est pas toujours reconnue par des guillemets ou la mention o in nom d'auteur.

II reprend aussi quelques-unes des remarques faites dans un article que $j$ 'ai publié il y a presque vingt ans («Macbeth et la tradition de la Moralité...», Caliban, $\mathrm{V}, 1968$ ), à la différence près qu'aujourd'hui je ne souhaiterais plus associer Macbeth aussi étroitement à la Moralité : certes, des images aussi bien textuelles que thêatrales ont une permanence à travers différents types dramatiques appartenant à la période post-médiévale anglaise, mais l'organisation dramatique et en particulier la conception du personnage ne sont plus dans les pièces de l' «âge d'or élisabethain» ou du début du règne des Stuart, ce qu'elles étaient à la fin du XVe ou dans les deux premiers tiers du XVIe siècle. Par ailleurs je serais bien incapable de dire aujourd'hui ce qu'est la «tradition de la moralité»!

Le titre que je viens d'annoncer «La confusion des signes», n'est qu' un des titres possibles qui s'étaient présentés à mon esprit : un autre titre, et c'est sur ce thème que je vous propose de débuter, pourrait être: «dédramatisons et redramatisons ! », apparemment sans rapport avec le précédent ; mais peut-être si en définitive. Je m'en explique. Dans un premier temps, donc, dédramatisons. J'emploie ce terme dans une acception, banale, familière, «triviale» comme disent les linguistes. Autrement dit : «n' en faisons pas un drame !» Ne faisons pas de Macbeth une «chute de l'homme», une «crucifixion», une «Descente aux Enfers» (allusion 
à certaine scène de la pièce sur laquelle nous aurons évidemment l'ocassion de revenir). Et aussi dure et sombre que soit l'action considérons que ce n'est qu'un jeu, sur le thêâtre, et dont la fin est le divertissement, non pas au sens pascalien du terme, qui est détournement du regard des problèmes essentiels, mais au sens d' «esbaudissement», «pleasant mirth», comme il est annoncé dans le sous-titre de la plupart des interludes moraux ou pas du XVIe siècle. N'oublions pas que les spectateurs avaient à choisir quand ils voulaient se divertir, entre un combat de coqs... et Macbeth, et que certains d'entre eux choisissaient Macbeth.

N'oublions pas non plus le caractère conventionnel de la forme théâtrale : les sujets ne sont pas tragiques en soi, mais c'est leur traitement qui est tragique. Des choses très sérieuses, et très voisines de ce qui est exprimé dans Macbeth, ont été dites sur une scène de thêâtre à l'aide de la comédie, puisque les interludes et autres pièces dites «morales» du XVIe siècle, sont en réalité des comédies dans leur forme. Le XVIe siècle est tout bruissant du débat humaniste sur les genres, et il serait bien étonnant que dramatruges et spectateurs en eussent été totalement innocents.

Truismes que tout cela, direz-vous ; mais je crois qu'il faut se répéter ces truismes inlassablement de peur de tomber dans le psychologisme et le moralisme naïfs. Il faut répéter qu'au thêâtre un espace aussi négligeable que l'épaisseur d'un cheveu peut séparer le tragique du comique : ne pensons pas seulement au texte, mais aussi à l'ensemble de la célébration théâtrale. Des scènes comme celles de l'apparition du glaive prophétique, ou celle des coups frappés à la porte, peuvent, à la faveur d'une grimace de trop ou d'une intonation mal placée, facilement basculer dans le comique. Si l'on y songe bien, les prophéties qui structurent l'action (et on connaît la valeur architectonique de ces tropes que sont les prolepses); celle selon laquelle Macbeth ne sera défait que par un adversaire non né d'une femme, ou si le bois de Bimam va à la rencontre du château de Dunsinane, ne sont en définitive que de "grosses ficelles» dont la résolution d'un point de vue réaliste, si l'on exclut le contexte et le verbe poétique, est indigne d'un roman policier moyen.

Examinons, si vous le voulez bien, ce que les contemporains pensaient de la tragédie. En 1599, la Tragédie paraît sur la scène de A Warning for Fair Women, participant au prologue de cette pièce, qui est un étrange mélange de techniques surannées et d'interrogations d'actualité. Elle engage avec la comédie et l'«histoire», (entendez par là, la pièce historique) une discussion qui ressemble plutôt à une querelle de harangères qu'à un débat académique. On y apprend que la tragédie ne supporte ni le violon (cats guts), musique favorite de la comédie, ni le tambour (sheepskin) de la pièce historique. (Peut-on en déduire que ce sont les bois qui lui fournissent sa musique caractéristique ?). Après cela la comédie brosse de la tragédie un portrait caricatural, mais non dénué de vérité où elle apparaît comme caractérisée (je cite en vrac) par le meurtre, l'apparition d'un spectre, la réalisation d'une vengeance, l'utilisation du brouillard artificiel et où il a été dit que son décor est constitué de tentures noires. Malgré ces caractéristiques plutôt lugubres le spectateur garde un œil vif et joyeux (cheerful) comme un enfant à qui on a promis qu'il irait voir les clowns'. 
Voilà donc les éléments matériels qui situaient telle représentation dans le camp de la tragédie ; non que la tragédie se limitât à ces quelques accessoires, mais ce sont là des signes qu'il ne faut jamais perdre de vue si on veut parler de thêâtre.

Et je m'aperçois que tout en dédramatisant, je me suis déjà engagé dans la re-dramatisation de mon sujet, c'est-à-dire sa réinsertion dans une perspective dramatique, et non plus seulement litttéraire. Et je voudrais examiner avec vous un certain nombre de signes dramatiques qui ont acquis droit de cité et valeur, par une longue présence dans la tradition théâtrale du XVIe siècle, et qui, je pense, apparaissent perturbés dans Macbeth. C'est de cette confusion que me paraît naître le tragique.

Sous 1'appellation «signe dramatique» je traiterai de ce qui pourrait se dénommer aussi «images, mais pas seulement des images textuelles, telles celles étudiées par Miss Mahood, C. Spurgeon... mais aussi des images situationnelles, fondées sur des éléments de décor ou des accessoirses, ou sur la mise en scène.

Pour moi, tout se révèle dans Macbeth à partir de la scène du Portier, qui est comme le diapason qui donne le ton sur lequel il faut interpréter le rapport de Macbeth avec les signes hérités du théâtre antérieur. Depuis l'article de Wickham (Sh. Survey, 1966), qui reprend une suggestion de W. Hales de 1884, nous savons que cette scène est largement inspirée de la scène de la Descente aux Enfers, présente dans la plupart des Cyles, et particulièrement développée dans ceux de Wakefield et d'York. En fait, cette scène provient, par une tradition ininterrompue, de 1'Evangile apocryphe de Nicodème ; un maillon essentiel dans la transmission étant un poème semi-dramatique, moyen-anglais «Harrowing of Hell». C'est ce poème qui, semble-t-il, invente le personnage du Portier, un portier déjà indigne de sa tâche, peureux, et donc comique, qui se fait rabrouer ainsi par le Christ :

Whare is he bat 3 ate ward?

Ich hold him for a coward

$143-4$.

Vision passagère dans le poème moyen anglais, le portier s'échappe tout de suite en courant. Arrive alors la confrontation solennelle du Christ avec les portes de l'Enfer

Helle 3ates, here y 3ou felle, And seppen will ich herwe helle

149-50.

Cette confrontation était d'autant plus présente à l'esprit des spectateurs qu'elle était renouvelée dans deux moments de la liturgie catholique : l'un qui revenait cycliquement à la fin de la procession de la fête des Rameaux, 1'autre plus 
exceptionnel lors de la Dédicace d'une église. Alors l'Evêque se présentait devant la porte fermée de l'église, et après qu'elle avait été heurtée trois fois par la croix processionnelle, l'évêque répétait à haute voix les paroles (apocryphes, certes, mais tout aussi impressionnantes que des paroles canoniques) Tollite Portas. Voilà ce que nous dit Wickham. On peut donc superposer notre scène du Portier à la scène correspondante des Mystères : Macbeth estle diable, Dunsinane l'Enfer, et Macduff le Sauveur ou son messager. On peut même ajouter pour poursuivre dans le sens de la littéralité, que, comme la descente aux enfers du Christ a eu pour effet la sortie des âmes des Prophètes et autres personnages de l'Ancien Testament, l'irruption de Macduff qui ouvre les portes de Dunsinane permet la fuite des Malcolm et de Donalbain qui assureront plus tard la succession légitime;

On peut ajouter aussi que l'intensité de l'allusion religieuse de la scène est renforcée du fait qu'elle est un maillon d'une chaîne d'allusions aux étapes principales de l'Histoire du Monde qui était représentée dans les Mystères : montée au Calvaire (Golgotha, I.ii. 41, «th' ingredience of our poison' $d$ chalicelTo our own lips, I. vii, 11) qui évoque à la fois «père détournez ce calice» du Christ mourant, et l'éponge remplie de fiel (poison) proposée par le soldat romain. De la descente aux enfers, la transition se fait aisément, selon l'habitude de l'exégèse typologique, à la vision du Jugement Dernier dont elle est une préfiguration. Ce glissement est signalé par l'évocation insistante de la trompette (II. iii. 82), à laquelle nous adjoindrons le tocsin (the alarum bell) autre signe avant coureur d'un cataclysme, puisque Lady Macbeth le réinterprète explicitement comme une trompette (II. iii. 82). Macbeth appelle Donalbain et Malcolm, et les invite à sortir du sommeil comme on sort d'une tombe :

\section{As from your graves rise up, and walk like sprites \\ II. iii. 80.}

image ambiguẽ, mais fondée sur celle du Jugement Dernier.

La fréquence des images religieuses nous invite à une interprétation chrétienne traditionnelle de Macbeth comme pécheur et de la pièce commel'histoire de son expiation. Mais est-ce aussi simple que cela ?

Nous situerons notre argumentation sur le terrain le plus sûr : celui de la représentation.

Dans Wakefield comme dans York c'est le Christ qui prononce la première tirade de la scène. A York il s'adresse à l'homme et rappelle le sens de la Passion et la promesse qu'elle accomplit. De même à Wakefield il évoque la rédemption. Il est donc seul en scène, puisqu'il souhaite que les âmes dans les Limbes aient connaissance de sa venue. Il se tient devant les spectateurs, et la porte de l'Enfer est derrière lui. A l'appel du Christ, les diables sont pris de panique et on peut imaginer qu'ils montrent le bout du nez ou poussent des hurlements par-dessus les créneaux du château d'enfer, dont la porte est constituée par la célèbre gueule d'enfer. C'est ensuite par cette même gueule que les âmes rachetées sortiront en procession au 
chant du Te Deum (Wakefield) et du Laus Tibi Domino (York) ${ }^{2}$.

Dans les mystères le spectateur est donc devant l'enfer. Son point de vue est donc celui du Christ venant heurter l'huis terrifiant. Pas plus que le Christ il ne pénétrera en enfer. Ses horreurs lui sont cachées. Cette vision est une vision optimiste, comme en font foi la joie d'Adam, d'Eve, etc. et les chants de louange qui s'élèvent de la procession qui se constitue alors. Dans Macbeth nous sommes de l'autre côté de la porte : le décor est inversé. Mais cette inversion du décor impliquet-elle qu'il y a un simple renversement de point de vue, les polarités restant inchangées : sommes-nous en enfer, et le Sauveur va-t-il venir frapper à la porte ? Tout le problème est là. Ici les citations de $D r F$ austusne sont pas très probantes, car il faut remarquer que selon toute vraisemblance cette dernière pièce est de près de 20 ans antérieure à Macbeth et il s'est passé bien des choses entre temps, non seulement en politique, mais aussi et surtout dans les esprits.

Certes la situation n'est pas brillante à l'intérieur de Dunsinane : un noble écossais a tué son roi pour usurper son trône : mais est-ce l'enfer pour autant ? Sommes-nous dans une Ecosse proto-historique où règneraient les terreurs primitives? sommes-nous dans quelque royaume théocratique médiéval où la légitimité divine du souverain serait incontestée ? ou dans l'Angleterre de 1606 ? Dans une perspective de la réception, il faut compter avecl'histoire, et j'ai été très sensible aux rappels historiques que nous a fait $M$. Dubu sur les conditions d'accessions au trône de Jacques I et ce que cette passation de pouvoirs avait pu signifier pour les Anglais qui en avaient été les témoins. Si la tempête de la nuit, «the night has been unruly» (II. iii. 55), rappelle le tremblement de terre du Vendredi Saint, ou une atmosphère de Jugement dernier, «the great doom» (II.iii. 79), il faut remarquer qu'elle sévit tout autant à l'extérieur qu'à l'intérieur du château. La dialectique intérieur/extérieur, équivalente à l'opposition protection/nature sauvage, danger, n'est ni inversée, ni respectée ; mais nous assistons plutôt à une égalisation, une dépolarisation.

On peut remarquer aussi la complication et la perturbation de l'opposition par auto-enchâssement de cette opposition intérieur/extérieur : en effet d'autres portes ont été ouvertes, fracturées dans ce cas, que celles du château de Dunsinane : celle du Temple oint du Seigneur (II. iii. 69) qu'on peut aussi traduire : «temple de l'oint du seigneur». La métaphore est filée, renforçant ainsi la présence de cette opposition :

and stole thence/ The life of the building

II. iii. 69-70.

Nous sommes en droit de poser la question : y avait-il donc un Temple au sein de l'enfer ? (Il est quelquefois bon de pousser les métaphores à bout, pour voir comment elles réagissent !).

Cette expérience nous montre que le rappel de la mise en scène traditionnelle, ou du symbolisme chrétien habituel ne nous conduisent pas jusqu'à la 
solution. A la différence d'Orphée ou du Christ lui-même, le spectateur a pu pénétrer en enfer. L'opposition entre extérieur/intérieur a perdu son sens : les ténèbres sont générales, comme nous le rappelait récemment J-M. Maguin.

Comme l'art de la communication est l'art de faire compliqué quand on pouvait faire simple, j'ai commencé mon étude par l'examen de la mise-en-scène et des métaphores, alors que j'aurais pu tout simplement écouter le Portier lui-même. Que ce Portier plaisante ou soit un peu pris de boisson n'est ni surprenant, ni significatif. Son ancêtre, le petit diable Ribald, chargé de la garde de la poterne de York et de Wakefield, contient en germe dans son nom même toutes les possibilités de clownerie et de bouffonnerie (les plaisanteries blasphématoires sont aussi vieilles que la religion et que les pièces religieuses : le Satan de Wakefield rabroue ce même Ribald en lui disant :

Hanged be thou on a cross (p. 379)

ce qui peut parâtre d'un goût douteux aujourd'hui.

Que dit donc le Portier?

Après un rappel ironique du rapprochement que le public avait déjà fait spontanément entre ces coups frappés à la porte et ceux frappés par le Christà la porte de l'enfer - rappel qui tient tout autant de la didascalie que du dialogue - le portier prononce le mot clé de son monologue qui est aussi le mot clé de la pièce entière : equivocator. Le monde qui est révélé à ce moment-là est un monde équivoque et trompeur. La scène du portier est un simple révélateur : nous l'avons vu, la simple présence des spectateurs du côté de l'enfer enfreignait les règles à la fois de la mise en scène médiévale et de la théologie. Cetteéquivoque est valable pour toute la pièce.

Cette équivoque a été mainte fois notée. K. Muir (introduc. et notes à l'Arden Sh.) entre autres. Mais j'aimerais essayer d'aller un peu plus loin que mes devanciers dans son analyse.

Lorsque Muir écrit :

The reference to treason... looks forward to the dialogue between Lady Macduff and her son, and to the long testing of Macduff by Malcolm - which shows the distrust and suspicion which grow from equivocation and hypocrisy...

et plus loin :

Macbeth's own equivocation, by an ironical twist, becomes merely an aspect of Truth. It is a brilliant counterpart to the equivocation of the fiend that lies like truth. 
il restreint beaucoup le problème et le fait dériver dangereusement vers le plan psychologique.

Qu'il y ait ou non une allusion topique dans la mention du «farmer» premier personnage évoqué par le Portier, le simple fait qu'il y ait obscurité et sens caché à découvrir dans les paroles du Portier, sont comme tous les passages comiques jouant sur le langage, des mises en auvre thêâtrales de la notion d'équivoque et du rôle trompeur des mots que Shakespeare dénonçait déjà en 1593 dans $L L L$ (et aussi dans $T N$ )

Je voudrais envisager la notion d'équivoque dans deux domaines où elle est traditionnellement employée : le domaine de la justice et celui du théâtre. «Equivocator» et son synonyme «ambidexter» est un terme qui désigne le juge ou le juré qui reçoit des pots de vin des deux parties à la fois et ainsi constitue un empêchement grave à la manifestation de la vérité. La justice - divine ou humaine - est très présente dans Macbeth. Elle y est décrite sous son aspect idéal : «even handed Justice» (I. vii. 10) mais, comme toujours, ce sont les bouffons qui, par leur cynisme, sont ceux qui approchent au plus près la vérité. La justice est présente dans le monologue du Portier par l'intermédiaire de la balance symbolique :

Here's an equivocator that could swear in both the scales against either scale.

$$
\text { (II. iii. 10) }
$$

La même notion est présentée un peu plus loin à l'aide du terme «neutral» qui est un autre équivalent technique de «equivocator» ou «ambidexter»; Ridley nous décrit en 1555 ce genre d'individu : «Theymay be called neutrals, ambidexters, or rather such as can shift on both sides». Cette acception de «neutral» est nécessaire à la compréhension de la phrase de Macbeth qui est construite sur une série d'oppositions :

Who can be wise, amaz'd, temperate and furious, Loyal and neutral, in a moment?

II. iii. 109.

En deuxième lieu «equivocator» et «ambidexter» sont des termes familiers des interludes du XVIe siècle. Ils y sont appliqués à ce personnage particulier (ou plus exactement cette fonction particulière) qui porte le nom de Vice, et qui a pour caractéristique de tourner son rôle de tentateur en comédie. Dans toutes ces «equivocations» du Portier à propos de la boisson et de ses effets sur l'urine et sur l'activité sexuelle, le courage et le sommeil (II. iii. 28-37), les spectateurs voyaient certainement l'affleurement de la fonction Vice avec laquelle ils étaient familiers. 
Si l'apparition du Portier est fugace, en revanche la présence de l'équivocation et de l'ambiguitté est générale, dans une pièce qui s'ouvre sur le refrain chanté à l'unisson par les trois sorcières: «Fair is foul, and foul is fain (I. i. 11 et I. iii. 38) où les batailles sont à la fois engagées et perdues (I. i. 4), toujours incertaines (doubtful, I. ii. 3), où le malheur vient de l'endroit d'où l'on attendrait le réconfort (I. ii. 27).

On pourrait multiplier les exemples ; mais cette fusion née de l'union de contraires a été trop souvent commentée pour qu'on insiste...

Ce qui a peut-être été moins exploré c'est la nature exacte de cette ambiguïté. Qu'est-ce que cette «ambiguïté morale» dont parlent certains critiques (Somerset, «Fair is foul... Eliz abethan. Theatre V, 1975). Ce n'est pas l'ambiguitté de l'indécision, mais plutôt l'ambiguité de l'indifférenciation ; traditionnellement le tentateur ne pousse pas sa victime à faire le mal, en lui présentant un choix moral clair, mais son argument est justement de le persuader que le choix en question n'existe pas, ou n'est pas pertinent tant qu'il y a de la vie. En effet l'action de Dieu, depuis la Création jusqu'au Jugement Dernier, consiste à séparer (la lumière des ténèbres, la terre des eaux, le bon grain de l'ivraie, les justes des réprouvés) à faire «le ménage» pour ainsi dire, à créer la valeur par l'opposition des contraires. Dans la période médiévale, qui s'étend loin dans le XVIe siècle, lorsque les vérités du dogme s'imposent encore à la majoritê, l'argument qui a toutes les chances d'entraîner l'adhésion au mal est celui de l'autruche : refuser d'envisager le choix, profiter du monde, être indifférent. «Reckless» est le maître mot qui donne la clé de l'état de celui qui vit dans le péchés. Après les soubresauts religieux et politiques qui ébranlent le dogme en minant ses bases institutionnelles (dans l'Eglise et dans l'Etat), en passantà une époque où les valeurs sont fondées sur des lois plus sociales que divines, la conséquence de la contravention aux lois n'est plus l'insouciance, mais l'angoisse, «the ravell' d sleeve of care», II. ii. 36). L'état dans lequel s'enfonce le contrevenant n'est plus l'indifférence mais l'indifférenciation, «confusion», (II. iii. 67$)^{4}$. L'homme est pris d'une sorte de vertige de la confusion d'avant la Création, de la nuit, des ténèbres, du brouillard et des esprits qui proviennent du brouillard(III. v. 24-7), du sommeil qui figure l'antithèse de la raison, raison qui symétriquement est engagée dans le même processus de laïcisation. Cet effondrement dans les abîmes de l'indiscernable est bien pire que la chute dans le péché, car pour cette faute il n'y a pas de rédemption, pour cette faute il n'y a que remord, mais jamais repentir, au sens où repentir est entendu comme le premier acte de la pénitence. Pour ce nouveau type de pécheur la confession est impossible (II. ii. 26-32). Il connaît au contraire la torture morale incessante «restless ecstacy» (III. ii. 22); son esprit est un nid de scorpions (III. ii. 36) et le salut («ther's comfort yet», III. ii. 39), le seul salut, c'est la fuite en avant (III. iv. 121, 135-6). Cet homme sait qu'il n'est qu'au début de son calvaire, qu'il a devant lui un long et dur chemin :

we are yet but young in deeds

(III. iv. 143). 
Privé de la raison divine, il cherche désespérément la raison humaine. Entre les deux c'est le noir : voilà la tragédie qu'illustre Macbeth (la pièce). Entre les deux c'est la déraison de Lady Macbeth c'est le règne des signes ambigus, des signes inversés : du sommeil, à la fois image de repos et image de mort, des apparences trompeuses, visages ou vêtements, de la nuit qui favorise mais en même temps dissimule le crime

that my keen knife see not the wound it makes

I. v. 12.

Lady Macbeth évoque, pour en renverser aussitôt le sens, la scène du sacrifice d'Isaac où Dieu arrête le bras du sacrificateur : scène importante, puisque image de la miséricorde divine, anticipation typologique de l'Incarnation et de la Rédemption. Ce «hold, hold», qui, selon la tradition chrétienne a arrêté le bras d'Abraham, lady Macbeth le refuse. C'est le même «hold» que Macbeth refuse avec le même entêtement au moment de sa mort.

Un deuxième exemple d'inversion des signes nous est fourni par la scène du Docteur : l'attente du spectateur est construite et orientée par son souvenir d'une scène identique favorite des pièces folkloriques, et qui s'est aussi introduite dans certains interludes, où le Docteur redonne vie à un héros exécuté rituellement au cours de la danse dite du «sabre». Cette origine folklorique est confirmée par la présence de la liste de médicaments et la mention des maladies qu'il convient de soigner : tout ceci évoque le boniment commun à l'«espicier», ou marchand d'herbes, des Passions continentales et au Docteur des pièces folkloriques anglaises. Le docteur du rituel folklorique effectue la guérison ; celui de Macbeth avoue au contraire son impuissance et ne songe qu'à fuir (V. ii. 75 et V. iii. 61-2).

Ces renversements, cette confusion, qui ont pour effet d'abolir le monde tel qu'il est en général représenté, ont bien de quoi troubler l'âme du spectateur, et ce spectacle répond bien aux buts que le personnage de Tragédie avait déclarés dans le Prologue de $A$ Warning.

I must have passions that must move the soule, Make the heart heave, and throb within the bosome, Extorting tears out of the strictest eyes...

Prol. 44-6

L'histoire de Macbeth est une histoire à faire frémir. C'est une histoire exemplaire de traitre, complète, avec tête du vaurien promenée au bout d'une pique, comme finissent tous les traîtres de mélodrame. C'est une histoire à ne pas raconter aux enfants, car elle leur donnerait des cauchemars ; $M$ acbeth est un cauchemar en 
soi. Mais peut-être n'est-ce qu'un cauchemar, c'est-à-dire un mauvais rêve, c'està-dire un récit décalé par rapport à un autre récit dans lequel il est enchâssé. L'histoire de Macbeth, qui est comme un exemplum inversé, commence par ce qui est donné explicitement comme appartenant au domaine du surnaturel et vient buter à la fin sur les acclamations faites au nouveau roi, «Hail» (V. ix. 20-4), et sur un monologue conclusif, conventionnel, en partie résumé, en partie prospectif, qui nous ramène à un plan sans mystère, une conclusion du genre : «ils seront heureux et ils auront beaucoup d' enfants» :

This and what needful else

That calls upon us, by the grace of Grace, We will perform in measure, time and place.

(V. ix. 37-9)

Macbeth est avant tout un exemplum, et cet exemplum raconte l'histoire d'un homme qui tue son roi par ambition ${ }^{5}$. Il n'est pas question d'aller a l'encontre du sens de surface. Mais, au-delà de l'ambition, cette histoire exemplaire est celle d'un dérèglement et d'une quête. Une histoire de noble écossais ne serait pas suffisante pour "soulever l'âme», si elle ne touchait pas d'une autre manière les spectateurs contemporains de Jacques 1er, se constituant ainsi en tragédie.

Ici aussi, je prendrai comme exemple un signe dramatique, après la scène du Portier, à la fin de l'acte II, Rosse rencontre un vieil Homme devant la porte du château de Dunsinane. Est-ce un effet de réel ? Ce vieillard représente-t-il la population avoisinante ? Cette explication apparaîtrait bien naïve.Je proposerai que ce vieillard est à la fois un observateur et un double. Observateur, car il n'appartient pas à la diégèse. Il rapporte, comme Lenox, la perturbation, la confusion qui s'est emparée du monde, mais avec plus de poids car il n'appartient pas à la suite royale. Il parle aussi à partir d'une position de vieillard, «three score and ten» (II. iv. 1) qui lui confere l'autorité. Sa conclusion est une bénédiction pour ses interlocuteurs et pour ceux qui :

Would make good of bad, and friends of foes

(41).

Il n'exprime pas là, le souhait vague que tout le monde «soit bien gentil», mais très précisément, que s'achève la confusion et qu'émerge un principe qui permette de dire quel est le bien, et quels sont les amis. Il appelle ainsi de ses voux une nouvelle Création qui séparerait la lumière des ténèbres. Il est une image divine (comme les personages de Mercy, ou de Peace, qui paraissent dans les interludes) mais aussi la figure humaine qui vit selon ces principes divins. Il est le double 
nostalgique de Macbeth, la figure mythique de l'homme d'avant l'époque de la confusion. Cette interprétation est coroborrée par un autre passage parfaitement explicite qui intervient après l'apparition de Banquo :

\author{
Blood hath been shed ere now, i' th'olden time \\ Ere humane statute purg'ed the gentle weal, \\ Ay, and since too, murthers have been perform'd \\ Too terrible for the ear: the time has been, \\ That, when the brains were out, the man would die, \\ And there an end; but now, they rise again, \\ With twenty mortal murthers on their crowns, \\ And push us from our stools
}

(III. iv. 74-81).

Nostalgie d'un temps où les choses étaient ordonnées : la faute était suivie de la rétribution et les morts étaient morts jusqu'au jour de la Résurrection. Pespectives illusoires, bien sûr, puisque toutes les époques ont eu leurs angoisses, mais il est naturel que les angoisses du présent s'expriment sur fond d'ordre passé. Cet Old Man est, de façon ironique, l'homme nouveau selon St Paul ; mais ce que représente Macbeth, c'est la quête d'un nouvel homme nouveau.

$\mathrm{Si}$, comme le propose C. Belsey (The Subject of Tragedy 1985) la notion de sujet émerge d'une pratique signifiante, Macbeth est 1'histoire de la recherche d'un nouveau sujet, que nous nommerons, à la suite de $\mathrm{C}$. Belsey, le «sujet de l' humanisme libéral». En effet, le savoir est au centre de ce drame.

Au début du jeu d'Adam, mystère français du XIle siècle, Dieu interpelle Adam : «Adam» - «Sire». Dieu poursuit : «Formé te ail de limo terre»; et Adam répond «Ben le sai». Adam sait, par la confiance qu'il a en la parole divine, par procuration. Macbeth ne sait pas, il veut savoir : au cœur des horreurs (Acte III, iv) il veut revoir les sœurs fatales pour savoir :

For now I am bent to know (III. iv. 133) (voir aussi I. iii. 70 et 78).

La quête du savoir représente la recherche de la cohérence du sujet énonciateur. Cette recherche est manifestée par plusieurs signes. D'abord la répétition lancinante du terme man, dont je ne ferai pas une recension détaillée. On peut dire en résumé que la gamme des acceptions de ce terme dans Macbeth se situe entre le sens médiéval de «homme perdu», parce qu'en proie à la colère sous la forme spécifique de la violence ${ }^{6}$. C'est ce sens qui paraît en particulier lorsque Macbeth s'adresse aux meurtriers qu'il a engagés pour assassiner Banquo. A l'autre extrémité, le terme Man figure l'être de conscience responsable de ses actions qui se dessine en creux tout au long de la pièce. L'être humain apparaît dans Macbeth comme 
discontinu : Malcolm est à la fois le sauveur de l'Ecosse, digne descendant de Duncan, et l'être le plus vicieux qui apportera à son pays des malheurs incommensurablement plus atroces que ceux provoqués par l'usurpateur. (IV. iii. 38-114). Macbeth lui-même est le point de convergence des contraires. L'enfant chéri du courage (Valour's Minion, I. ii. 19), l'époux de Bellona (I. ii. 55) devient le meurtrier, le réprouvé (II. iii. 91-6; V. iii. 22-8). Il évoque lui-même la discontinuité du moi :

Who can be wise, amaz'd, temperate and furious, loyal and neutral, in a moment?

II. iii. $108-9$

Si Macbeth affirme justement, dans une déclaration si emphatique, son «single state of man» (I. iii. 140), c'est que cette unité n'est pas réalisée.

La formulation de cette recherche de l'unité passe par la forum stoïcienne (et dont l'histoire chrétienne est très longue), «connais-toi toi-même», plusieurs fois employée au cours de la pièce (II. ii. 72 ; IV. ii. 19). Elle sert maintenant de véhicule à l'idée d'autonomie du sujet. Le docteur dit de l'aliénation symbolique de Lady Macbeth,

therin the patient/Must minister to himself

(V. iii. 45-6).

Cet apprentissage de la liberté passe par l'expérience du libre arbitre ; là aussi l'histoire est longue. Le libre arbitre catholique est le privilège qu'a l'homme de faire le bien volontairement, mais il est dangeureusement voisin de l'abus que l'homme peut faire de cette même liberté en obéissant à sa fantaisie personnelle quand il n'est plus en accord avec la volonté divine? ${ }^{7}$. Sous l'influence calviniste, le terme Freewill prend de façon systématique une connotation négative. Les personnages de Freewill sont dans de nombreuses pièces, des viveurs et des chenapans. Chez Macbeth la volonté se manifeste sous forme d'un entêtement qui s'exprime à l'aide du verbe modal will, répété dans la dernière scène: «I will not yield» (V. ix. 27) et «yet I will try the last « (id. 32).

Le moi insaisissable et malheureux est représenté par le bateau naufragé qu'évoquent les sorcières. Je renvoie ici à l'analyse si révélatrice de Y. Peyré «Macbeth et le naufrage ${ }^{8}$ qui insiste sur l'image non seulement du navire, mais sur celle encore plus pertinente de ce qu'il appelle l'anti-navire, le tamis, qui est aussi à la Renaissance emblème de la sagesse, puisque c'est l'instrument de la séparation du bien et du mal, de la clarification, qui est objet de regret et de désir, mais qui est paradoxalement attribué dans Macbeth aux sorcières. Si l'objet même de la raison est confié aux agents de la confusion, le désordre n'en est que plus menaçant encore ! 
L'expression de la quête du moi passe enfin par l'utilisation dramatique des sorcières et autres apparitions surnaturelles. Nous les considérerons comme des signes dramatiques de la confusion. Comme tous les gens dramatiques ils ont deux référents : un référent théâtral, et un référent dans le monde. La tradition du théâtre à personnages allégoriques est là pour nous assurer que le public acceptait sans broncher que des personnages allégoriques, mais à statut hybride, car représentant à la fois des catégories théologiques (la Miséricorde) et des qualités humaines (la miséricorde de tel ou tel roi), puissent cohabiter et dialoguer avec des personnages représentant des humains. Ces différences de niveaux de représentation ne posaient donc aucun problème. La scène du Harrowing of Hell est elle-même comme le montre très clairement le Ludus Conventriae, une scène allégorique encartée dans le récit de la Passion, autre changement de niveau auquel le public s'attendait.

Avec la Renaissance le surnaturel «laïque» remplace peu à peu les allégories morales, probablement parce que le référent dans le monde de ces dernières était de moins en moins sûr, alors que sorcières et fantômes étaient au centre de la curiosité et faisaient l'objet d'études para-scientifiques.

La fameuse question : Art thou / A dagger of the mind, (II. i. 37-8) exprime à l'évidence un doute à propos du référent dans le monde. Le référent thêâtral ne peut en aucun cas être en cause. Quand il entre en scène, la première parole de Macbeth «foul and fair», reprenant la déclaration des sorcières, montre que leur discours n'avait été que la manifestation dramatique de l'intériorité humaine : c'était là un rappel utile de la «convention théâtrale» ${ }^{9}$. Mais ensuite, à l'intérieur même de cette convention les personnages de la pièce se livrent à une mise en doute de la légitimité de ces représentations (à la différence de Hamlet qui ne met jamais en doute l'existence du spectre de son père). La pièce de Macbeth porte tout autant sur la nature des apparitions que sur la culpabilité du personnage Macbeth, qui n'est pour moi que le héros d'un exemplum encarté.

L'interrogation sur la nature du surnaturel, comme l'autre interrogation qui lui est intimement liée, sur la modalité d'existence du sujet sont présentées sous forme de débats. Joël Altman nous rappelle avec insistance et raison l'importance de l'héritage rhétorique dans la tradition théâtrale Tudor.

Nombre d'interludes ne sont que des débats plus ou moins avoués, plus ou moins déguisés. Dans les pièces plus tardives, cette influence se retrouve dans la façon dont les arguments contradictoires sont développés, et dont l'issue de la pièce reste ouverte sans véritable conclusion. Macbeth en est un exemple frappant.

Voyez comment Banquo argumente (à propos des sorcières) :

Were such things here, as we do speak about, Or have we eaten on the insane root That takes the reason prisoner? 
Voyez comment $\mathrm{M}$. raisonne à propos des révélations des sorcières :

This supernatural soliciting...

Je ne peux pas tout citer.

(I. iii. 130))

Voyez les if qu'emploie Macbeth, comme pour désigner une action fictive, comme on le fait dans les démonstrations logiques :
If it were done...
If th' assassination...
(I. vii. 1)
(id. 2)

Macbeth etLady Macbeth délibèrentcomme dans un effort désespéré pour se prouver qu'ils sont maîtrres de leur destin (I. vii. 28-83). Nous avons déjà vu comment Macbeth pèse le pour et le contre à propos de la réalité du poignard (II. i. 33-61).

Le caractère logique et quelquefois ratiocinant de certains monologues ne doit pas nous cacher que les problèmes débattus sont sans cesse des problèmes existentiels, à propos desquels il est sans doute inévitable en fin de compte de faire «un drame» (aux deux sens du terme). Et, parodiant le titre de Altman, The Tudor Play of Mind, je vous proposerai de voir Macbeth, comme The Stuart Tragedy of Mind. 


\section{NOTES}

1 How some damnd tyrant, to obtaine a crowne,

Stabs, hangs, impoysons, smothers, cutteth throats,

And then a Chorus too comes howling in,

And tels us of the worrying of a cat,

Then of a filthie whining ghost,

Lapt in some fowle sheete, or a leather pelch,

Comes skreaming like a pigge halfe stickt,

And cries Vindicta, revenge, revenge :

With that a little Rosen flasheth forth,

Like smoke out of a Tobacco pipe, or a boyes squib :

Then comes in two or three like to drovers,

With taylers bodkins, stabbing one another,

Is not this trim? Is not here goodly things?

Warning for Fair Women, Prologue, 50-63.

Tragedy (s'adressant à la Comédie et à l'Histoire) :

Are you both gone so soone? Why then I see

All this faire circuite here is left to me :

All you spectators, turne your chearfull eie,

Give intertainment unto Tragedie...

Ibid. 91-4.

2 Les coups frappés à la porte ne rappellent pas seulement la scène de la Descente aux Enfers : ils s'inscrivent aussi dans une tradition comique, comme le prouve le passage de Fulgens and Lucres de Medwall:

(Knocking at the door)

A A, there commyth one -I here hym knoke.

He knokythe as he were wood!

One of you go loke who it is.

B Nay, nay, all the meyny of them iwis can not so moche gode.

A man may rappe tyll this naylis ake Or ony of them wyll the labour take

To gyve hym an answere.

II F\&L, 73-80.

3 Hécate le sait bien qui déclare que

...Security

Is mortals' chiefest enemy

III. v. 32-3.

Security n'est pas exactement «over confidence» comme l'explique K. Muir (note au v. 32), mais plus techniquement «recklessness».

4 Voir : 
(I) Shall raise such artificial sprites

As, by the strength of their illusion

Shall draw him unto his confusion

III. v. 27-9

Hécate utilise ici confusion dans le double sens de «damnation» et de «situation de brouillage», «confusion d'avant la Création du monde».

5 Dans les manuels pénitentiels et moraux du moyen-âge, l'ambition est présentée comme une branche de l'orgueil. La présence insistante des images vestimentaires introduit dans Macbeth une référence permanente à l'orgueil. De plus c'est bien dans $M$ qu'on trouve l'expression : vaulting ambition. Lisons tout le passage :

To prick the sides of my intent, but only ...I have no spur

Vaulting ambition, which o'erleaps itself

And falls on th' other

I. vii. $25-8$

La note de K. Muir, là non plus ne sera pas d'un grand secours. (a-t-on jamais vu un cavalier se servir de ses éperons pour sauter en selle ?)Il s'agit de la représentation bien connue du «Cavalier Orgueil», qui, présumant de ses forces est désarçonné par son cheval, et tombe à terre (Médaillons au Portail du Sauveur de la cathédrale d'Ámiens et au Porche Sud de Chartres); on trouve Orgueil sur un cheval boîteux (donc prêt à tomber) dans Li Tornoiement Antecrit de Huon de Mery (c. 1235) ; voir H.A. Tardy, thèse de 3ème cycle, 1985, Amiens, p. 118-23.

6 Hécate décrit Macbeth comme «wrathful», III. v. 12.

7 Ici encore c'est Hécate qui dit la vérité sur Macbeth. Lorsqu'elle l'appelle a wayward son (III. v. 11). Wayward signifie ici «qui fait un mauvais usage de son libre arbitre»; voir O.E.D., s. V. wayward ; «self-willed, capriciously wilful». Exemple : «A, thou generacioun unbyleeful and weiward» (traduc. de Wiclif de Matthieu xvii, 16 ; la Vulgate porte perversa (de vertere : qui s'est détoumé du droit chemin).

8 Cahiers du CERLI, 3, Toulouse, 1981.

9 Il faudrait rappeler ici comment les persomnages allégoriques ont laissé graduellement la place à des personnages «réalistes» au cours du XVIe siècle, pour se réfugier en fin de compte dans les prologues, épilogues et dans de petites scènes occasionnelles, qui font figure de «cadre». Dans ce cadre ils côtoient des personnages surnaturels (fantômes, fées, magiciens...) à partir des années 1570 ; voirJames $I V$, Friar Bacon and Friar Bungay, Midsummer Night's Dream...). 